\title{
Analysis of Query Dependent Multi-Document Summarization using using Feature based and Cluster based Methods
}

\author{
Prof. S. T. Kolhe \\ Asst. Professor, Information Technology, \\ Sanjivani College of Engineering, Kopargaon \\ Prof. Y. S. Deshmukh \\ Asst. Professor, Information Technology, \\ Sanjivani College of Engineering, Kopargaon
}

\begin{abstract}
WWW World Wide Web is the largest source of information. The huge amount of information is available on internet. The problem is to get the exact data or relevant information from the source data. In the past years great amount of work has been done on text document summarization but that is over the single document. So the important issue is to summarize the data from Multi-Document, and that is the challenge over document summarization. Single document summary seems to capture both the information well but it has not been the case for multi document summary where the overall comprehensive quality in presenting informative summary often lacks. It is found that most of the existing methods tend to focus on sentence scoring and less consideration is given to the contextual information content in multiple documents. In this study, some survey on multi document summarization approaches has been presented. We will direct our focus notably on two well-known approaches to multi document summarization namely the feature based method, cluster based method. With the aim of enhancing multi document summarization, specifically news documents, a novel type of approach is outlined to be developed in the future, taking into account the generic components of a news story in order to generate a better summary.
\end{abstract}

Keywords - Document Summarization, Summarization, Single Document, Multi Document, Summarization Approaches, Cluster and clustering techniques. Feature based method.

\section{INTRODUCTION}

The need of text summarization has recently increased due to the proliferation of information on the Internet. With the availability and speed of internet, information search from online documents has been eased down to user's finger tips. However, it is not easy for users to manually summarize those large online documents. For example, when a user searches for information about earthquake which occurred in Killari, Maharashtra (India), and the user will probably receive enormous articles related to that event. The user would definitely opt for a system that could summarize those articles. The goal of text summarization is condensing the source text into a shorter version preserving its information content and overall meaning. The objective and approach of summarization of documents explain the kind of summary that is generated. For example, it could be indicative of what a particular subject is about (closely related to a user query), or can be informative about what the whole content of document is all about. Besides that, approach towards text summarization can be either extractive or abstractive. In extractive type summarization, important sentences are identified and directly extracted from the original document, i.e. the final summary consists of original sentences. On the other hand, in abstractive type summarization the sentences which are selected from the original document are further processed to restructure them before concatenating them into final summary. This process usually involves deep natural language analysis and sentence compression.

\section{SUMMARIZATION}

\subsection{SUMMARY}

"An abbreviated, accurate representation of the content of a document preferably prepared by its author(s) for publication with it." Such abstracts are also useful in access publications and machine-readable databases (American National Standards Institute Inc., 1979)[2]. By understanding the type of summary i.e., indicative, informative, extractive and abstractive, we can then apply them to either single document or multi document. This study focuses mainly on informative and extractive type multi document text summarization. The distinct characteristics that make 
multi document summarization rather different from single document summarization is that multi document summarization problem involves multiple sources of information that overlap and supplement each other, being contradictory at occasions. So the key tasks are not only identifying and coping with redundancy across documents, but also ensuring that the final summary is both coherent and complete[12]. The contributions of this study can be summarized as follows: We discuss the four notable approaches of multi document summarization and present it with related research from literatures. The benefits and limitations concerning these approaches are also discussed. At the end of this study, a novel type of approach is outlined to be developed in the future, for news documents summarization. We aim to incorporate the generic components of a news document in order to generate a better summary [12]. The rest of the study is organized as follows: First we present the survey on four multi document summarization approaches namely the feature based method, cluster based method, graph based method and knowledge based method. Then we outline the proposed multi document summarization method; i.e., the component based method. Finally we end with conclusion. Daniel Marcu [3] in his PhD thesis dwells on the discourse structure of natural language texts citing from important work done by Grosz and Sidner[4] and Mann and Thompson[5]. He argues that rhetorical relations between elementary textual units hold important keys to finding the relation between various units of a text. While relying on a tree like structure for finding the abstract structure of a text, Marcu explores the use of cue phrases in not only finding the structure of a text but also to evaluate the rhetorical relations between them. These rhetorical relations can be used to identify the importance of various textual units, hence their importance in text summarization. Using such rhetorical relations the most important parts in a discourse text can be found and some partial ordering can be enforced on them to select the vital parts of the text. Lin and Hovy [7] describe an approach to identify topic signatures present in text for automatic summary or for information retrieval purpose. Their approach uses a pre-classified corpus for training and thus has its own limitations. However their focused use of topic signatures for producing summaries outperforms the base lining method and tf-idf methods for extracting topicrelevant sentences for summary. Zajic Lin and Dorr [9] incorporated a multi-document sentence trimmer into a feature based summarization system. They used trimming to pre-process documents and create multiple partially trimmed sentences as alternatives for the original sentence. The count of trimming operations done is then used as a feature in the sentence ranker. Ideally the trimmed sentences should be grammatically correct. Syntactic trimming offers three distinct advantages; firstly, a net increase in the average number of sentences per summary; second, removal on non-relevant constituents; and third, more space is created for adding relevant sentences. Error analysis has shown that while sentence compression is making space for additional sentences, more work is needed in the area of generating and selecting the right candidates. Conroy and Schlesinger [10] mention in their submission to DUC 2006, their modified approach for sentence splitting and sentence trimming. They remove the use of POS tagger for the sentence splitting and instead choose a conservative sentence trimming strategy which relies on a list of function words. The trimming is very conservative and the error analysis showed an error rate of less than $3 \%$ that is less than three percent of the input sentences were made ungrammatical by this trimming task. The summarization approach discussed by Jing and McKeown [9] is based on statistical methods. Initially the most important sentences are extracted from the source text. The extracted sentences are then joined together by analyzing their discourse structure and modifying them as required. The preprocessing step includes the following:

$>$ Sentence reduction: Removal of extraneous phrases.

$>$ Sentence combination: Combination of sentences based on the context.

$>$ Syntactic transformation: Altering the grammatical structure of the sentences as required.

$>$ Lexical paraphrasing: Replace phrases with their paraphrases

$>$ Reordering: The selected sentences are then reordered to make the overall summary comprehensible. Summarization of document by clustering approach is another very used concept

\section{MULTI DOCUMENT SUMMARIZATION}

The approaches followed for document summarization is classified in many ways:

1) Abstractive Vs Extractive:

Abstraction involves a more in-depth analysis of the source document, condensation of its information content and generation of a summary which is cohesive and appears as if it was written by a human. At the same time, it should be able to satisfy the information need. This requires more sophisticated techniques and computational power[1][2]. Extraction techniques, on the other hand, focus on the most important in the document and perceive it in the form of words, clauses or sentences on the surface level. Complete understanding of the semantic and syntactic of the source document is not necessary. This requires lesser computational power and hence, it is more suitable for generating onthe-fly summaries. 


\section{2) Multi-Document Vs Single-Document:}

Most of the early summarization systems were Single document summarizers. When used to summarize multiple documents which discuss about a similar topic (For example, several news articles pertaining to an event), they would process each of them individually and the resulting summaries would contain a considerable amount of repeated information as there is repetition in the source articles DUC 2005. Whereas, a multi-document summarizer treats the whole set of documents as a single document representing a common topic. This way, the summaries will not contain repeated information.

3) Query dependent Vs Query independent:

Query dependent systems focus on summaries which are influenced by the query. The query is also analyzed semantically and provided as an input to the system. The summary generation process is guided by the information contained in the query[1][2].Multi document summarization approaches: A number of research study have addressed multi document summarization in academia (Erkan and Radev, 2004a, Wan and Yang, 2008, Haribagiu and Lacatusu, 2010) and illustrated different types of approaches and available systems for multi document summarization. In this study we direct our focus notably on four well known approaches to multi document summarization. Our discussion will be based on the following pattern: For each method, we will first discuss its main idea. Following that, we will look at some research study from related literatures. Finally the benefits and limitations concerning each method are commented. Feature based method: Extractive type summarization involves identifying the most relevant sentences from the text and put them together to create a concise summary. In the process of identifying important sentences, features influencing the relevance of sentences are determined. Here we list some of the common features that have been considered for sentence selection. Word frequency: The idea of using word frequency is that important words appear many times in the document. The most common measure widely used to calculate the word frequency is tf and idf. Title/headline word: Occurrence of words from the document title in sentence indicates that the sentence is highly relevant to the document. Sentence location: Important information in a document is often covered by writers at the beginning of the article. Thus the beginning sentences are assumed to contain the most important content. Sentence length: Very short sentences are usually not included in summary as they convey less information. Very long sentences are also not suitable to represent a summary. Cue word: There are certain words in a sentence which indicate that the sentence is carrying an important message in the document (e.g., "significantly", "in conclusion"). Proper noun: Sentences containing proper noun representing a unique entity suchlike name of a person, organization or place are considered important to the document.

\section{FEATURE BASED METHOD}

Our first approach to Multi document summarization is feature based Summarization.

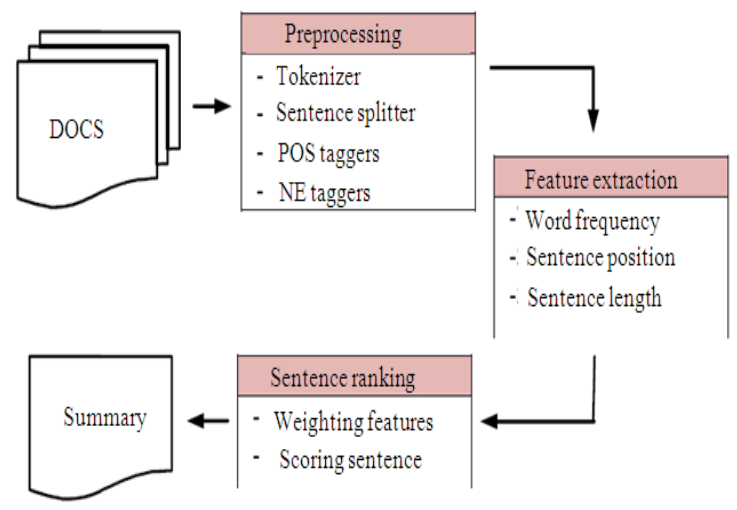

Fig. 4.1- feature based summarizer architecture.

Let us show a simple example for scoring a sentence S. Consider that we select three features[12]

namely title word, sentence length and sentence location. Calculation of the score for each of these features as in (Suanmali et al., 2009) is shown below:

No. title word in $\mathrm{S}$

Title Word, $\mathrm{T}=\mathrm{No}$. word in title 
No. word in S

Sentence Length, $\mathrm{L}=\overline{\text { No. word in longes sentence }}$

Sentence position, $\mathrm{P}=5 / 5$ for 1 st, $4 / 5$ for 2 nd, $3 / 5$ for $3 \mathrm{rd}, 2 / 5$ for 4 th, $1 / 5$ for 5 th, $0 / 5$ for other sentences. After computing each of the feature score, the total scoring of a sentence is given by Eq. 1:

Score_Si $=\mathrm{w}_{1} \mathrm{~T}_{\mathrm{i}}+\mathrm{w}_{2} \mathrm{~L}_{\mathrm{i}}+\mathrm{w}_{3} \mathrm{P}_{\mathrm{i}}$

where, Score Si is the total score of sentence Si. The terms Ti, Li and Pi are the feature scores of sentence Si based on the title words it contains, sentence length and sentence position respectively. w1, w2 and w3 are the weights for the linear combination of the three features. Fig.4.1 depicts the generalized architecture of a feature based summarizer. We can see that text features score are combined for sentence scoring, as shown in Eq. 1. But not all text features are treated with same level of importance as some of the features have more importance or weight and some have less. Thus emphasis should be given on dealing with the text features based on their importance. This issue can be overcome by using weight learning method. Many researchers have been using various weight learning methods in their study. Binwahlan et al. (2009) introduced a novel text summarization model based on swarm intelligence technique known as Particle Swarm Optimization (PSO). The feature scores were adjusted using the weights resulting from the training using the PSO. The training set consist pairs of document and human reference summary. They implemented the sentence scoring using the following Eq. 2:

$$
\operatorname{Score}(\mathrm{S})=\sum_{\mathrm{i}=1}^{5} \mathrm{w}_{\mathrm{i}} \times \text { Score_f } \mathrm{f}_{\mathrm{i}}(\mathrm{S})
$$

where, Score (S) is the score of the sentence $\mathrm{S}$, w1 is the weight of the feature i produced by PSO, $\mathrm{i}=1-5$ showing that 5 text features where used and Score fi(s) is the score of the feature i. The use of PSO for optimization have also been proven to be robust in other domains as well (Nacy et al., 2009, Balaji and Kamaraj, 2011). Another weight learning approach was described by Bossard and Rodrigues (2011) who approximated the best weight combination using a genetic algorithm for their multi document summarizer. By using the genetic algorithm, a suitable combination of feature weights can be found. Table 1 lists some of the weight learning methods applied to text summarization.

\section{CLUSTERING METHOD}

The idea of clustering is to group similar objects into their classes. As far as multi documents are concerned, these objects refer to sentences and the classes represent the cluster that a sentence belongs to. By looking at the nature of documents that address different subjects or topics in the documents, some researchers try to incorporate the idea of clustering into their study. Using the concept of similarity, sentences which are highly similar to each other are grouped into one cluster, thus generating a number of clusters. The most common technique to measure similarity between a pair of sentences is the cosine similarity measure where sentences are represented as a weighted vector of tf-idf[2]. Once sentences are clustered, sentence selection is performed by selecting sentence from each cluster. Sentence selection is then based on the closeness of the sentences to the top ranking tf-idf in that cluster. Those selected sentences are then put together to form the final summary Fig. 5.1. 


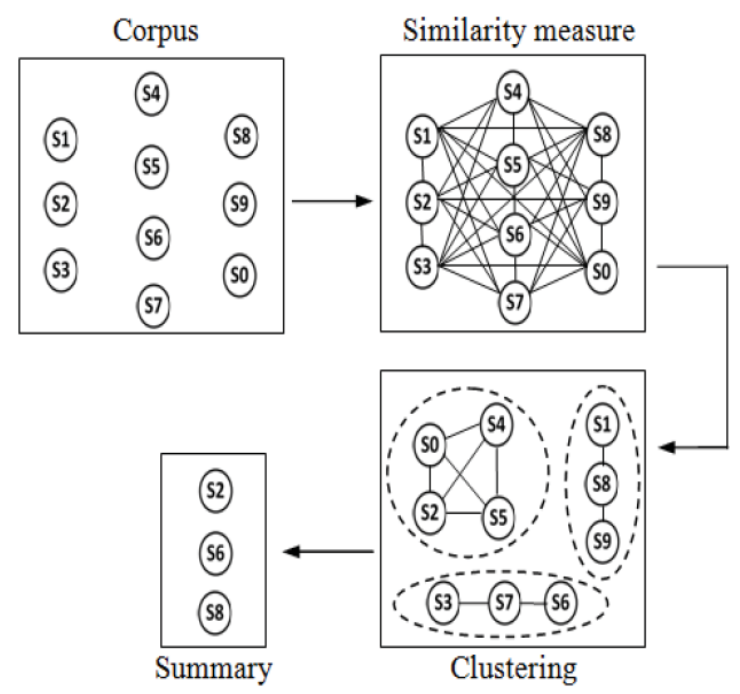

Fig.5.1 cluster based Summarization

The term "clustering" is used in several research communities to describe methods for grouping of unlabeled data[12]. These communities have different terminologies and assumptions for the components of the clustering process and the context in which clustering is used. Thus, we face a dilemma regarding the scope of this survey. The production of a truly comprehensive survey would be a monumental task given the sheer mass of literature in this area. The accessibility of the survey might also be questionable given the need to reconcile very different vocabularies and assumptions regarding clustering in the various communities.
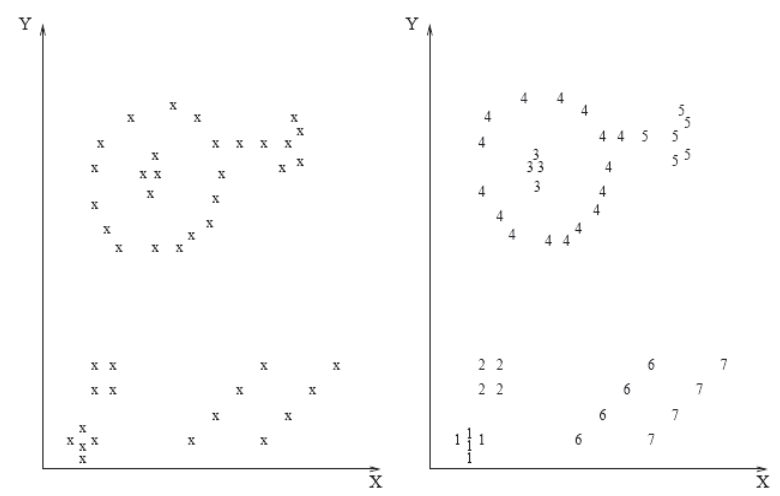

Fig.5.2 Data Clustering

a) Monothetic vs. polythetic: This aspect relates to the sequential or simultaneous use of features in the clustering process. Most algorithms are polythetic; that is, all features enter into the computation of distances between patterns, and decisions are based on those distances. A simple monothetic algorithm reported in Anderberg [1973] considers features sequentially to divide the given collection of patterns.

b) Hard vs. fuzzy: A hard clustering algorithm allocates each pattern to a single cluster during its operation and in its output. A fuzzy clustering method assigns degrees of membership in several clusters to each input pattern. A fuzzy clustering can be converted to a hard clustering by assigning each pattern to the cluster with the largest measure of membership. 
c) Deterministic vs. stochastic: This issue is most relevant to partitional approaches designed to optimize a squared error function. This optimization can be accomplished using traditional techniques or through a random search of the state space consisting of all possible labelling.

d) Incremental vs. non-incremental: This issue arises when the pattern set to be clustered is large, and constraints on execution time or memory space affect the architecture of the algorithm. The early history of clustering methodology does not contain many examples of clustering algorithms designed to work with large data sets, but the advent of data mining has fostered the development of clustering algorithms that minimize the number of scans through the pattern set, reduce the number of patterns examined during execution, or reduce the size of data structures used in the algorithm's operations.

\section{IMPLEMENTATION}

Module 1: Clustering of the Documents:

Clustering Algorithms:

K-means clustering algorithm

By using two different approaches of clustering K-means algorithm will be used to form Cluster i.e. Hierarchical and Partitional algorithm.

Algorithms are as follows;

1. Agglomerative Hierarchical K-means

2. Nearest Neighbour Clustering Algorithm

K-Means algorithm will be used to form the related cluster.

Module 2: Creating the document graph.:

Each cluster becomes a node in the document graph. The document graph $G(V, E)$ of a document $d$ is defined as follows:

- $d$ is split to a set of non-overlapping clusters $t(v)$, each corresponding to a node $v \in V$.

- An edge $e(u, v) \in E$ is added between nodes $u, v \in V$ if there is an association between $t(u)$ and $t(v)$ in $d$.

Hence, we can view $G$ as an equivalent representation of $d$, where the associations between text fragments of $d$ are depicted.

Module 3: Adding Weighted Edges to the Document document graph:

Graph :The following input parameters are required at the pre computation stage to create the

1. Threshold for edge weights. Only edges with weight not below threshold will be created in the document graph. (A threshold is user configurable value that controls the formation of edges) [2][3].

2. Minimum text fragment size. This is used when a fragment is too long, which would lead to large nodes (text fragments) and hence large summaries. Users typically desire concise and short summaries. Adding weighted edge is the next step after generating document graph. Here for each pair of nodes $u, v$ we compute the association degree between them, that is, the score (weight) EScore $(e)$ of the edge $e(u, v)$. If Score $(e) \geq$ threshold, then $e$ is added to E. The score of edge $e(u, v)$ where nodes $u, v$ have text fragments $t(u), t(v)$ respectively is:

$$
\text { EScore }=\quad \frac{\operatorname{size}(\mathrm{t}(\mathrm{u}))+\operatorname{size}(\mathrm{t}(\mathrm{u}))}{\sin }
$$

where $t f(d, w)$ is the number of occurrences of $w$ in $d$,

$\operatorname{idf}(w)$ is the inverse of the number of documents containing $w$, and

size $(d)$ is the size of the document (in words). That is, for every word $w$ appearing in both text fragments we add a quantity equal to the $t f i d f$ score of $w$. Notice that stop words are ignored. 


\section{Module 4: Adding Weight To Nodes In Document}

Graph :When a query $\mathrm{Q}$ arrives, the nodes in $\mathrm{V}$ are assigned query-dependent weights according to their relevance to Q.Adding Weight to Nodes In Clustered Document Graph When a query $Q$ arrives, the nodes in $V$ are assigned query-dependent weights according to their relevance to $Q$. In particular, we assign to each node $v$ corresponding to a text fragment $t(v)$ node score $N \operatorname{Score}(v)$ defined by the Okapi formula as given below.[1]

$$
\operatorname{NScore}(v)=\sum_{i \in Q . d} \ln \frac{N-d f+0.5}{d f+0.5} \cdot \frac{\left(k_{1}+1\right) t f}{\left(k_{1}(1-b)+b \frac{d l}{a v d l}\right)+t f} \cdot \frac{\left(k_{3}+1\right) q t f}{k_{3}+q t f}
$$

tf is the term"s frequency in document,

qtf is the term"s frequency in query,

$\mathrm{N}$ is the total number of documents in the collection,

$\mathrm{df}$ is the number of documents that contain the term,

$\mathrm{dl}$ is the document length (in words),

avdl is the average document length and $\mathrm{k} 1$ (between 1.0-2.0), b (usually 0.75), and k3 (between 0-1000) are constants.

\section{CONCLUSION}

This study provides a general survey on multi document summarization approaches. Indeed, this study has been tailored in a way that researchers whom are new to the area of text summarization can grasp the idea of various multi document summarization approaches. Four types of approaches have been discussed, namely the feature based method, cluster based method. It appears that each of these methods possess its own advantages towards multi document summarization. At the same time, there are some issues or limitations pertaining to those methods. For future improvement, we propose a novel approach, taking into account the generic components of a news story in order to generate a better summary which is well suited for an informative type summary generation. We belief that the proposed component based approach can alleviate some of the aforementioned limitations.

\section{REFERENCES}

[1] A.K. Jain ,M.N.Murty and P.J.Flynn. Data Clustering: A Review.

[2] Deshmukh Y.S.,Shirole B.S.prof. Pankaj Dhshore "Analysis of Query dependent Summarization Using Clustering Techniques". IJCTEE Volume2, Issue 1.2012.

[3] H. Daumé and D. Marcu. Bayesian query-focused summarization. In Proceedings of COLING-ACL2006.

[4] Yogan Jaya Kumar and ,Naomie Salim “Automatic Multi Document Summarization Approaches” Journal of Computer Science 8: 133140, 2012 ISSN 1549-3636 @ 2012 Science Publications.

[5] S. Agrawal, S. Chaudhuri, and G. Das. DBXplorer: A System For Keyword-Based Search Over Relational Databases. ICDE, 2002

[6] Mann and Thompson. Rhetorical Structure Theory: A theory of text organization. Technical Report ISI/RS-87-190, Information Sciences Institute, 4676 Admiralty Way, Marina del Rey, California 90290-6685, June 1987.

[7] Chin-Yew Lin, Eduard Hovy. The automated acquistion of topic signatures for text summarization, Proceedings of the $18^{\text {th }}$ conference on Computational linguistics - Volume 1, July 2000.

[8] R. Barzilay and M. Elhadad: Using lexical chains for text summarization. ISTS, 1997.

[9] D.M. Zajic, B. Dorr, J. Lin, R. Schwartz, Sentence Compression as a Component of a Multi-Document Summarization System , Proceedings of the Document Understanding Conference, 2006.

[10] Conroy, J., Schlesinger, J., O'Leary, D., \& Goldstein, J. Back to basics: CLASSY 2006. In Proceedings of the 2006 document understanding conference (DUC 2006) at HLT/NAACL2006, New York, NY, 2006.

[11] H.H. Chen, J.J. Kuo, and T.C. Su: Clustering and Visualization in a Multi-Lingual Multi DocumentSummarization System. ECIR, 2003

[12] Yogan Jaya Kumar and 2 Naomie Salim "Automatic Multi Document Summarization Approaches “ Journal of Computer Science 8 (1): 133-140, 2012ISSN 1549-3636 ㄷ 2012 Science Publications. 